\title{
EL APOYO FAMILIAR Y LA ADHERENCIA EN EL TRATAMIENTO DE HIPERTENSIÓN ARTERIAL
}

\author{
FAMILY SUPPORT AND \\ ADHERENCE IN THE TREATMENT \\ OF HYPERTENSION \\ Recibido 20 de mayo 2014 \\ Aceptado agosto 2014
}

Correspondencia:

M. en C. Adela Alba-Leonel Antiguo camino a Xochimilco y Viaducto Tlalpan,

Col. San Lorenzo Huipulco C. P. 14370

Tlalpan, México, D. F.

Tel. 55562332 Ext. 269

adelaalbaleonel@yahoo.com.mx

\section{Autores:}

M. en C. Adela Alba-Leonel

Dr. Guillermo Fajardo Ortiz

Ing. Joaquín Papaqui Hernández

Palabras clave: Hipertensión arterial, apoyo familiar y adherencia al tratamiento.

Key Words: Hypertension, family support and adherence to treatment. 
RESUMEN

Introducción: La hipertensión arterial es un factor de riesgo para las enfermedades cardiovasculares; es un problema de salud que se incrementa cuando el paciente no cuenta con apoyo familiar.

Objetivo: Conocer cómo influye el apoyo familiar en el cumplimiento o adherencia del tratamiento de hipertensión arterial.

Metodología: Se realizó un estudio transversal en pacientes hipertensos que acudieron al Instituto Nacional de Cardiología (INClCh) de la ciudad de México, la recolección de la información fue a través de un cuestionario ex profeso que contempló dos secciones una para los pacientes y otro para los familiares de los pacientes.

Resultados: Se estudiaron 234 pacientes hipertensos del INCICh de más 20 años de edad, el 64\% son casados, el $47.7 \%$ cohabita con su cónyuge y el 33\% con su hija(o); el 88\% tiene una familia nuclear; 137 (56\%) son pacientes descontrolados (47\% del género femenino y 57\% masculino) 103 (44\%) controlados (54\% del género femenino y $46 \%$ masculino). Con respeto al apoyo familiar el $84 \%$ vive con un familiar y sólo el $79 \%$ está en contacto diario con el paciente, en cuanto al apego farmacológico, el 56\% le recuerda que debe tomar el medicamento, el 82\% de la administración del medicamento la realiza el paciente mismo y el 76\% le ayuda a su familiar a entender las indicaciones del médico. Las variables de apoyo familiar en las que se encontró una asociación estadísticamente significativa fue que el hipertenso debe medirse la tensión arterial en la casa y debe llevar su dieta y cuando el enfermo no la sigue lo amenaza de los riesgos que implica.

Discusión: El apego al tratamiento se vincula de forma directa con el apoyo que los familiares le otorguen al enfermo.

Conclusiones: El éxito del tratamiento depende fundamentalmente del apego, sin embargo el apoyo familiar juega un papel importante.

Palabras clave: Hipertensión arterial, apoyo familiar y adherencia al tratamiento.

\section{ABSTRACT}

Introduction: Hypertension is a risk factor for cardiovascular disease, is a health problem that increases when the patient has no family support.

Objective: To examine the influence of family support in the compliance or adherence of the treatment of Hypertension.

Methodology: A cross-sectional study was conducted in hypertensive patients who attended the National Institute of Cardiology ( $\mathrm{NClCh}$ ) in Mexico City, gathering information was through a questionnaire that expressly included two sections one for patients and one for relatives of patients.

Results: 234 hypertensive patients INCICh over 20 years old were studied, $64 \%$ were married, $47.7 \%$ cohabiting with their spouse and $33 \%$ with his daughter(0), $88 \%$ have a nuclear family, 137 (56\% ) are uncontrolled patients (47\% female and $57 \%$ male ) 103 (44\%) controlled (54\% female and $46 \%$ male). With respect to family support, $84 \%$ live with a family and only $79 \%$ is in daily contact with the patient, in terms of drug addiction, 56\% reminds you to take medication, $82 \%$ of the drug is performed by the patient and $76 \%$ helps your family understand the doctor's instructions. Family support variables in which a statistically significant association was that hypertension should measure blood pressure at home and you must take your diet and when the patient does not follow the threat of the risks found.

Discussion: Adherence to treatment is linked directly with the support that you grant the family sick.

Conclusions: The success of treatment depends mainly on the attachment, but the family support plays an important role.

Keywords: Hypertension, family support and adherence to treatment.

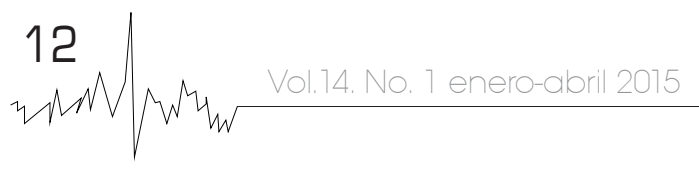




\section{INTRODUCCIÓN}

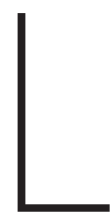

a Hipertensión Arterial (HTA) es una de las primeras causas básicas de mortalidad y el principal factor de riesgo para padecer accidente cerebro vascular, enfermedad cardiovascular y renal que también son causa de muerte en México ${ }^{3}$. Las complicaciones de la HTA se relacionan directamente con la magnitud del aumento de la tensión arterial y el tiempo de evolución.

Socialmente la hipertensión es considerada como una de las enfermedades más peligrosas e invalidantes; se le conoce como "la bailarina de los cuatro salones" por la repercusión en los órganos (corazón, cerebro, riñón y grandes vasos), por lo que representa uno de los grandes desafíos de la salud pública en el mundo. Así mismo, las enfermedades cardiovasculares también constituyen la primera causa de muerte en los países desarrollados y en muchos países del tercer mundo 4.5 .

De acuerdo con los datos de la Encuesta Nacional de Salud (ENSANUT) realizada en el 2006 6.7 se reportó una prevalencia de HTA del 30.7\%, donde el 12.5\% fue identificado por diagnóstico y el 18.2\% por las cifras de Tensión Arterial (TA). Para el 2012, la prevalencia se ha mantenido prácticamente sin cambios de acuerdo con los resultados de la ENSANUT 2012 ${ }^{8}$, donde se observa un mayor porcentaje de hipertensos que conocen su condición, lo que refleja una mejor detección. El 73\% de los individuos con diagnóstico previo de hipertensión, reportó estar en tratamiento farmacológico y el 76\% reportó tomarse la presión arterial al menos una vez al mes. Así mismo, en el 2012, se reportó que 22.4 millones de adultos padecen HTA, de los cuales 11.2 millones contaban con diagnóstico y de estos 8.2 millones se encontraban bajo tratamiento; sin embargo solo 5.7 millones están controlados?.

La hipertensión arterial es una enfermedad cuya prevalencia se encuentra en ascenso y que puede derivar en complicaciones, especialmente en los sujetos con un mal control de las cifras de TA4 5, 6. La elevación de la TA no produce de manera habitual manifestaciones clínicas, por lo que el paciente puede tener la impresión de que su enfermedad se encuentra controlada cuando en realidad no es así. ${ }^{10}$

Por lo anterior, es necesario tener un adecuado control de las cifras de TA en los pacientes hipertensos, con el propósito de aumentar su esperanza y calidad de vida ${ }^{17.12}$ y así mismo disminuir la morbilidad y mortalidad por hipertensión arterial.

Una de las principales causas del fracaso en el control de la tensión arterial es la falta de adherencia o apego al tratamiento, en la cual se pueden incluir varios factores como: el sistema de salud, factores socioeconómicos, tipo de tratamiento, características del paciente así como la percepción y conocimiento de su enfermedad. El apoyo familiar ${ }^{13}$ donde se: incentive las actitudes personales positivas en el monitoreo y control de la salud, favorezca la comunicación familiar para brindar auxilio - ayuda en momentos de crisis, se fomente los cuidados de la salud en general como: cuidados en su dieta, ejercicio físico y adherencia o apego al tratamiento antihipertensivo. Es importante señalar que la sola presencia de la familia junto al enfermo es capaz de favorecer la tranquilidad, fuerza, lo que lo hace sentirse seguro, protegido - amparado y lo incentiva a buscar soluciones para mejorar su bienestar, así como disminuir sus cifras tensionales ${ }^{14}$.

En vista de los expresado, es necesario desarrollar estrategias para determinar cuáles son los factores relacionados, sobre todo por que el paciente percibe su enfermedad como un factor que atenta contra la estabilidad de su familia, porque el nivel de adherencia al tratamiento puede declinar cuando existen cambios en la estructura y apoyo de la familia con respecto al paciente hipertenso ${ }^{15.16 .17 .}$ 


\section{METODOLOGÍA}

S e realizó un estudio descriptivo transversal, con un muestreo no probabilístico, la unidad de observación fueron los pacientes hipertensos que acudieron a consulta externa cardiológica del Instituto Nacional de Cardiología Ignacio Chávez (INClCh) de la Ciudad de México, durante el primer semestre del 2009, tomando en cuenta los siguiente criterios de inclusión: pacientes con diagnóstico de hipertensión arterial, con otra enfermedad concomitante, de 20 años de edad y más; que son atendidos en la consulta externa cardiológica; criterios de exclusión: pacientes de consulta externa de otros servicios, que no tuvieran un diagnóstico de hipertensión arterial, menores de 20 años de edad o que no aceptaron participar y como criterio de eliminación: pacientes que no proporcionaran la información.

Para la recolección de datos se diseñó un cuestionario ex profeso que contempló dos secciones una para los pacientes y otro para los familiares de los pacientes.

El cuestionario de los pacientes contemplo: datos generales del paciente, antecedentes heredofamiliares, antecedentes personales no patológicos, antecedentes patológicos con una sección específica sobre hipertensión arterial y somatometría.

Para la sección de los familiares se utilizó un cuestionario ex profeso validado por la Universidad de Guanajuato y Universidad la Salle Campus Guanajuato. El cual estuvo compuesto por: características de la Familia: Tipo de familia, tamaño (número de integrantes de la familia), corresidencia con los hijos, contacto con hijos, frecuencia de contacto y la comunicación no presencial con los hijos; en cuanto al apoyo se evaluó en la ministración, alimentación, indicaciones médicas, motivación, compañía ${ }^{18}$.
La información se capturó en Excel y se analizó con el Statistical Package for the Social Sciences, se realizó un análisis descriptivo.

En cuanto a los aspectos éticos, se les explicó a los pacientes mediante conocimiento informado que su participación era voluntaria, anónima y que la información se manejaría únicamente de forma general y con fines estadísticos.

\section{RESULTADOS}

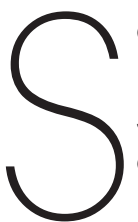
e estudiaron 234 pacientes hipertensos del INCICh de más 20 años de edad, el 64\% son casados, el $47.7 \%$ cohabita con su cónyuge y el 33\% con su hija (0); el 88\% tiene una familia nuclear, 137 (56\%) son pacientes descontrolados (47\% del género femenino y 57\% masculino) 103 (44\%) controlados (54\% del género femenino y $46 \%$ masculino).

Con respeto al apoyo familiar el 84\% vive con un familiar y sólo el 79\% está en contacto diario con el paciente, en cuanto al apego farmacológico, el $56 \%$ le recuerda que debe tomar el medicamento, el 82\% de la administración del medicamento la realiza el paciente mismo y el 76\% le ayuda a su familiar a entender las indicaciones del médico.

En cuanto al análisis pareado con respecto al apoyo familiar se observó que existe una asociación estadísticamente significativa para algunas variables como: apoyo para que el paciente se tome la tensión arterial en casa con una Xi= 7.5 y $p=0.024$

Aún cuando el control de las cifras de tensión arterial resulta indispensable para aumentar la esperanza y calidad de vida del hipertenso, este no se lleva a cabo por los familiares ya que estos hacen muy poco o nada para que se mida la tensión arterial en su casa o en otro lugar. Ver tabla I

\section{4 LMN NWhw Vol.14. No. 1 enero-abril 2015}


Tabla I. Apoyo familiar para que los pacientes hipertensos del INClCh, se midan la tensión arterial en su casa u otro lugar

\begin{tabular}{|c|c|c|c|}
\hline $\begin{array}{l}\text { Apoyo familiar para } \\
\text { que se mida la TA }\end{array}$ & $\begin{array}{c}\text { HTA } \\
\text { Controlado }\end{array}$ & $\begin{array}{c}\text { HTA } \\
\text { No controlado }\end{array}$ & Total \\
\hline Nunca & 16 & 7 & 23 \\
\hline Rara vez & 5 & 3 & 8 \\
\hline Ocasionalmente & 4 & 15 & 19 \\
\hline Casi siempre & 17 & 8 & 25 \\
\hline Siempre & 43 & 52 & 95 \\
\hline Total & 85 & 85 & 170 \\
\hline
\end{tabular}

Que el familiar lo apoye en llevar su dieta y que cuando el enfermo come más alimentos de los indicados lo amenaza con los riesgos que implica como: le recuerda el riesgo que significa no seguirla, le retira los alimentos, le insiste que le puede causar un daño, lo amenaza o simplemente no le dice nada; con una $\mathrm{Xi}^{2}=14.48 \mathrm{p}=0.006$, otras de las variables que influyen es que el familiar le ayude a entender la indicaciones médicas de cómo y cuándo debe de tomarse cada uno de los medicamentos, ya que en ocasiones no son legibles o entendibles las indicaciones médicas plasmadas en la receta médica.

\section{DISCUSIÓN}

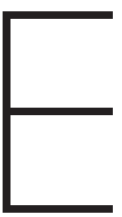

s indudable como influye el apoyo familiar en el tratamiento de la hipertensión arterial, ya que si esta contribuye positivamente o bien asume el rol de cuidador, se puede percibir como una respuesta satisfactoria donde se puede observar una disminución en las cifras de tensión arterial, en la morbilidad, complicaciones e inclusive la muerte ${ }^{19}$. En caso contrario donde la familia solo le proporcione preocupaciones familiares, esto generara estrés y ansiedad lo que hace que aumente sus cifras de TA o genere estados que lo lleven a reducir su autocuidado, como la falta de adherencia al tratamiento; observándose un mayor descontrol de la hipertensión arterial y ocasionando así complicaciones.

Se ha reportado que la "Armonía en la familia", "Mejoría financiera en la familia" y "Bienestar" mejora el "control de la presión arterial", así mismo, influye la dinámica familiar, el saber que alguien se preocupa por mí, en el alcance del control de la enfermedad de acuerdo con estudios cualitativos de la percepción del paciente en relación a los cuidados necesarios para el tratamiento de la

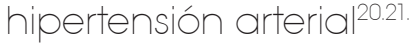

Por otro lado también reportaron que la "Preocupación de los hijos/nietos", "Relaciones con familiares/ amigos", "Alteración del estado de salud" y "Alteración emocional" son causas del aumento de su tensión arterial, la falta de autocuidado o adherencia al tratamiento referencias negativas 22.23 .

Así mismo la muerte de familiares puede generar en el enfermo crónico sentimientos de pérdida y sufrimiento, contribuyendo más para el descuido con su salud'?

El apoyo familiar, orientado a colaborar en el control de la presión arterial del hipertenso constituye una estrategia y un desafío para lograr la adherencia en el tratamiento ya sea farmacológico y no farmacológico. La consulta de enfermería en el control de las enfermedades crónico degenerativas es una oportunidad, para que el paciente, exprese sus sentimientos, dudas y se involucre a los familiares de los pacientes hipertensos, ya que en ocasiones estos no tienen conciencia de su importante papel en el control de la hipertensión ${ }^{24.25}$

No se puede lograr las modificaciones en el estilo de vida del paciente sin la participación de la familia, así como ayudar al paciente a entender las indicaciones del médico y mejorar la adhesión al tratamiento farmacológico. 


\section{REFERENCIAS BIBLIOGRÁFICAS}

1. Arrivillaga M., Cáceres R.D. , Correa D., Holguín L.E., Varela M.T., ÕPuede una intervención biopsicosocial reducir los niveles de presión arterial Med UNAB. 2006; 9(1): 20-27.

2. Causas de Mortalidad en México 2010. Estadísticas de Mortalidad, Secretaría de Salud. http://www.ssa. gob.mx (Consultado: 1 de febrero de 2014).

3. Stevens G, Dias R, Thomas K, Rivera J, Carvalho N, Barquera S. Characterizing the epidemiological transition in Mexico: National and subnational burden of diseases, injuries, and risk factors. PLoS Med 2008;5(6): e125.

4. OPS, Health in the Americas, 2002 Vol. I Washington DC PAHO; 2002 (PAHO Scientillc and technical Publication No 587)

5. Wetzel WJ, Silveira MOT. Hipertensão arterial: un problema de todos. Revista Nursing 2005; 81(8):70-5.

6. Gustavo Oláis, Juan Rivera, Teresa Shamah, Rosallba Rojas, Salvador Villalpando, Mauricio Hernández, Jaime Sepúlveda. Encuesta Nacional de Salud y Nutrición 2006: 1-131.

7. Barquera S., Campos N. Ismael, Hernández B. L., Villalpando S., Rodríguez G. C., Durazo A. R. , Aguilar S. C.A. Hypertension in Mexican adults: results from the National Health and Nutrition Survey 2006. Salud Publica Mex 20102010 Vol 52:Supl I S63- S71.

8. Secretaría de Salud, México. Instituto Nacional de Salud Pública, Encuesta Nacional de Salud 2012, Resultados Nacionales Síntesis ejecutiva, México, D.F., 2012:1-36.

9. Campos-Nonato I, Hernández-Barrera L, RojasMartínez R, Pedroza-Tobías A, Medina-García C, Barquera S. Hipertensión arterial: prevalencia, diagnóstico oportuno, control y tendencias en adultos mexicanos. Salud Publica Mex 2013;55 supl 2:S144-S150.

10. Figueroa-Castrejón $G$. Recordando al enemigo silencioso: La hipertensión arterial. Med Intern de Mex 1995; 11:79-82. (Links)

11. Chávez-Domínguez R, Vega-Estens $P$, Larios-Saldaña A. La hipertensión arterial y otros factores de riesgo coronario en la atención del primer nivel. Arch Inst Cardiol Mex 1993; 63:425-434 (Links)

12. Mearns $B$. Hypertension. Tackling challenges in the diagnosis and management of hypertension in 2012. Nat Rev Cardiol 2012;9(5): 253-254.

13. Gusmão JL, Mion D Júnior. Adesão do tratamento: conceitos. Rev Bras Hipertens 2006; 13(1):23-5.

14. Urizzi F. Vivências de familiares de pacientes internados en terapia intensiva: o outro lado da internação. (dissertação). Ribeirão Preto (SP): Escola de Enfermagem de Ribeirão Preto/ USP; 2005.

15. Marín R.F., Rodríguez M.M., Apoyo Familiar en el apego al tratamiento de la hipertensión arterial esencial; Rev. Salud Pública de México; Vol. 43, No. 4, julio-agosto de 2001: 336-339.

16. Louro B.I., Modelo de salud del grupo familiar, Rev Cubana Salud Pública; 2005;31(4) 05

17. Orueta S.R., Estrategias para mejora la adherencia terapéutica en patologías crónicas, Información terapéutica del Sistema Nacional de Salud, Toledo, Esp.; Vol.29 No.2:2005:40-48

18. Álvarez M. D., Evaluación del apoyo familiar, conocimiento sobre la Diabetes mellitus, dieta, su adherencia y su relación con el control glicémico en

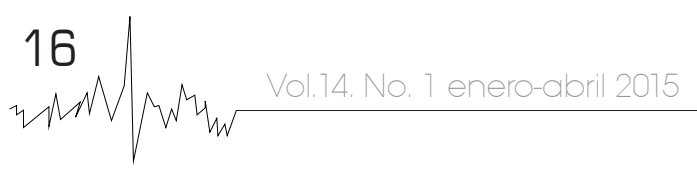




\section{REFERENCIAS BIBLIOGRÁFICAS}

pacientes diabéticos, Tesis para obtener el grado de especialista en Medicina Familiar, Universidad de Colima, Instituto Mexicano del Seguro Social, 2005: 135, Colima, Col.

19. Morales N.A.G., Redes de Apoyo Familiar y la salud del adulto mayor, Costa Rica; Trabajo Ïnal de graduación presentado en la Escuela de Estadística para optar por el grado de Master en Población en Salud, 2006: 1-55.

20. Costa RS, Nogueira LT. Contribución familiar en el control de la hipertensión. Rev Latino-am Enfermagem 2008 setembro-outubro; 16(5)

21. Marques S, Rodrigues RA, Kusumota L. O idoso após acidente vascular cerebral: alterações no relacionamento familiar. Rev Latino-am Enfermagem 2006; 14(3):364-71.

22. Silva LF, Guedes MVC, Moreira RP, Sousa ACC. Doença crônica: o enfrentamento pela família. Acta Paul Enfermagem 2002; 15(1):40-7.
23. Radovanovic CAT, Waidman MAP, Pereira MO, Felipes L, Ferrari IG, Marcon SS. Caracterizando os problemas de saúde e o cuidado domiciliar oferecido às famílias do projeto $\neq$ Promovendo a vida na Vila Esperança. Acta Scientiarum. Health Sciences 2004; 26(1):27-34.

24. Zapata S.M.A., El paciente hipertenso en la consulta de enfermería, Rev. Electrónica semestral de Enfermería; Enfermería Global; Administración, Gestión y Calidad, No. 9, noviembre 2006; Murcia, España; www.um.es/esglobal/9/09d03.html, consultado 28 febrero 2014

25. Guía de Hipertensión arterial en la consulta de enfermería; wWw.sescam.jccm.es/web/gaptalavera/ prof-guias/GAPTR-ConsEnf-guiahipertensionarterial. pdf. consultado 28 febrero 2014 